\title{
Factitious Hypoglycemia Caused by a Unique Pattern of Drug Use: A
}

\section{Case Report}

\author{
Mojtaba Akbari, ${ }^{1}$ Akbar Soltani, ${ }^{2}$ Mohammad Reza Mohajeri-Tehrani, ${ }^{2}$ Hamidreza Aghaei Meybodi, ${ }^{2}$ \\ Imaneh Sadat Mousavi Fakhr, ${ }^{2}$ and Sayed Mahmoud Sajjadi-Jazi ${ }^{2,}$ \\ ${ }^{1}$ Students' Scientific Research Center, Tehran University of Medical Sciences, Tehran, Iran \\ ${ }^{2}$ Endocrinology and Metabolism Research Center, Endocrinology and Metabolism Clinical Sciences Institute, Tehran University of Medical Sciences, Tehran, Iran \\ "Corresponding author: Sayed Mahmoud Sajjadi-Jazi, Endocrinology and Metabolism Research Center, Endocrinology and Metabolism Clinical Sciences Institute, Tehran \\ University of Medical Sciences, Tehran, Iran. Tel: +98-9133258106, E-mail: mahmood.sajadi@gmail.com
}

Received 2017 April 14; Revised 2017 October 08; Accepted 2017 November 13.

\begin{abstract}
Introduction: Factitious hypoglycemia, caused by the surreptitious use of insulin and sulfonylureas, is one of the most challenging differential diagnoses of hypoglycemia. Diagnosis is usually established via exclusion with respect to the special patterns of plasma insulin and C-peptide during hypoglycemic episodes.

Case Presentation: We report a case of recurrent hypoglycemic episodes and confusing patterns of insulin and C-peptide levels. In the primary evaluations, insulinoma was suspected considering the high plasma concentrations of insulin and C-peptide, besides negative urine and plasma sulfonylureas during hypoglycemic episodes. Considering the normal imaging studies and refractory hypoglycemia to medical therapy, distal pancreatectomy was performed. The patient had no episodes of hypoglycemia after the surgery. Five months later, similar episodes recurred. Further investigations revealed different plasma concentrations of insulin and C-peptide in each hypoglycemic episode. Regarding various biochemical patterns during hypoglycemia and absence of evidence supporting other differential diagnoses, we suspected factitious causes. Close observation revealed that the patient had a history of intermittent glyburide consumption and analog insulin injection.

Discussion: Most commercial insulin immunoassays can only detect human insulin and lack the ability to identify synthetic analog insulin. In addition, common detection methods for sulfonylureas and meglitinides are of low diagnostic value in the human plasma and urine. These laboratory defects can lead to the misdiagnosis of insulinoma or noninsulin-mediated hypoglycemia due to the surreptitious use of insulin secretagogues or analog insulin, respectively. Therefore, due to the lack of any definitive laboratory findings, clinical suspicion is the best strategy for diagnosis.
\end{abstract}

Keywords: Factitious, Hypoglycemia, Insulin, Glyburide

\section{Introduction}

Hypoglycemia is uncommon in nondiabetic people and may have different etiologies. Factitious hypoglycemia, resulting from the surreptitious use of insulin and sulfonylureas, is one of the most challenging differential diagnoses of hypoglycemia $(1,2)$. Diagnosis of factitious hypoglycemia due to insulin self-administration is established considering the inappropriately high plasma level of insulin in combination with low plasma C-peptide level during hypoglycemic episodes (3).

Sulfonylurea consumption produces a biochemical pattern, mimicking insulinoma (inappropriately high plasma levels of insulin and C-peptide), which can be diagnosed with positive drug screening (2-4). Herein, we present a patient with several episodes of hypoglycemia and confusing patterns of insulin and C-peptide levels.

\section{Case Presentation}

A previously healthy 46-year-old man referred to our hospital (Shariati hospital, Tehran, Iran) because of the onset of frequent symptomatic hypoglycemic episodes during the day, with a blood glucose level below 40 $\mathrm{mg} / \mathrm{dL}$ (measured on a glucometer). He had a history of rheumatic fever in childhood and alcohol consumption in social gatherings; however, he denied any alcohol consumption before the onset of the symptoms.

The patient was not on any medications, and his family history was unremarkable, except for diabetes mellitus in his mother, for which she was using metformin and glyburide. He worked for an elevator company and had received some medical training in youth. The physical examination was normal, and the 72-hour fasting protocol was applied for the patient. The insulin-secreting tumor was 
suspected considering the high plasma concentrations of insulin and C-peptide in symptomatic hypoglycemia (Table 1).

Roche Elecsys insulin assay is the selected insulin assay at our hospital. The patient's blood and urine samples were collected during a hypoglycemic episode and sent to a clinical laboratory in Iran, where liquid chromatographytandem mass spectrometry (LC-MS) was applied for sulfonylurea detection; the results were found to be negative (Table 1). At the end of the fasting test, the plasma glucose level increased more than $25 \mathrm{mg} / \mathrm{dL}$ on the glucagon test. The cortisol level was $22.8 \mu \mathrm{g} / \mathrm{dL}$.

To localize the tumor, imaging studies were performed. Abdominal computed tomography (CT), magnetic resonance imaging (MRI), and endoscopic ultrasonography (EUS) did not show any abnormal lesions. The patient was scheduled for exploratory laparotomy due to lack of selective arterial calcium stimulation tests at Iranian hospitals and persistence of symptomatic hypoglycemia despite treatment with diazoxide $(1200 \mathrm{mg} /$ day $)$ and dextrose $10 \%$ in water $(100 \mathrm{~mL} / \mathrm{h})$.

Intraoperative sonography was normal, and finally, distal pancreatectomy was performed. Histopathological examination of pancreatic tissues revealed no evidence of nesidioblastosis or insulinoma. The patient experienced no episodes of hypoglycemia after the surgery. Nonetheless, similar episodes recurred after 5 months. The plasma concentrations of insulin and C-peptide were different in each hypoglycemic episode (Table 1), suggesting endogenous hyperinsulinism (high insulin and C-peptide levels) versus noninsulin-mediated hypoglycemia (low insulin and C-peptide levels). The plasma and urine sulfonylureas were negative in all episodes (Table 1).

The imaging studies were repeated. Spiral CT scan and MRI of the abdomen only showed postoperative changes. The EUS demonstrated multiple small hypoechoic lesions (maximum size, $8 \mathrm{~mL} \times 7 \mathrm{~mL}$ ) in the head and body of the pancreas, suggesting multiple insulinomas, which further complicated the patient's clinical course. Because of a history of major surgery, EUS-guided fine-needle aspiration of the pancreatic lesion was performed before proceeding to any other surgeries; however, it only revealed blood.

With regard to the normal results of previous imaging and pathological studies, the new EUS finding was interpreted as a postoperative change (adenopathy) after consultation with an EUS operator and the patient's previous surgeon. There was no evidence of an insulin-like growth factor II (IGF-II)-secreting tumor on the laboratory or imaging studies. Because of frequent hypoglycemia, medical treatment was gradually initiated. The hypoglycemic episodes recurred several times throughout the day despite treatment with dextrose $10 \%$ in water $(100 \mathrm{~mL} / \mathrm{h})$, di- azoxide (1200 mg/day), octreotide (300 $\mu \mathrm{g} /$ day), and prednisolone (40 mg/day).

Considering various biochemical patterns during hypoglycemia and lack of evidence supporting other differential diagnoses, we suspected factitious causes. Frequent history-taking from the patient and his family members, including his wife and children, did not reveal any psychiatric problems, and they denied any conflicts. Incidentally, we discovered that the patient had asked one of his roommate's visitors to buy him glyburide. The serum and urine levels of sulfonylureas were assessed in each hypoglycemic episode, which became positive (Table 1).

After obtaining permission from the ethics committee and notifying the nursing supervisor, in a secret search of the patient's room, we found an insulin pen (NovoRapid) hidden in the water closet. Close observation revealed that the patient had intermittent glyburide consumption and analog insulin injections (insulin aspart or insulin glargine); however, he denied any drug use.

Finally, an interview with the patient's mother revealed that he had a marital conflict and had produced hypoglycemic symptoms to attract attention. The patient received several psychiatric consultations. After psychiatric and forensic pathology confirmation, he was discharged with quetiapine $(25 \mathrm{mg} /$ day) and outpatient psychiatric visits. However, four months later, he was found unconscious in a hotel room and died in spite of cardiopulmonary resuscitation. The exact cause of his death is unidentified.

\section{Discussion}

Hypoglycemia is rare in nondiabetic adults and may have different causes, such as drug use, critical diseases, cortisol deficiency, autoimmune hypoglycemia, IGF IIsecreting tumors, insulinoma, nesidioblastosis, and factitious causes. Factitious hypoglycemia is a very challenging diagnosis. The plasma levels of insulin and C-peptide can help differentiate this condition from other causes (5). Overall, diagnosis is quite challenging, as most commercial insulin immunoassays only detect human insulin and lack the power to detect synthetic analog insulin (6). In addition, common methods for sulfonylurea and meglitinide detection in the human plasma and urine are of low diagnostic value (7).

Most commercial insulin assays have low crossreactivity with insulin analogs (8). The detection power of a test for insulin analogs depends on both the type of platform and complexity of the analog. Some platforms, such as Roche Elecsys insulin assay, show more than $80 \%$ cross-reactivity with human insulin, while they exhibit 
Table 1. The Patient's Labratoary Data During Symptomatic Hypoglycemic Episodes ${ }^{\mathrm{a}}$

\begin{tabular}{|c|c|c|c|c|c|}
\hline & Date & Insulin $(\mu \mathbf{I} \mathbf{U} / \mathbf{m L})$ & C-Peptide (ng/mL) & BS $(\mathrm{mg} / \mathrm{dL})$ & Urine and Plasma Sulfonylureas \\
\hline \multirow[t]{3}{*}{ First admission (preoperative) } & January 6, 2016 & 15 & 2.4 & 28 & Negative \\
\hline & June 2, 2016 & 0.2 & 0.35 & 41 & Negative \\
\hline & June 8, 2016 & 117.9 & 10.5 & 48 & Negative \\
\hline \multirow[t]{3}{*}{ Second admission (postoperative) } & June 11, 2016 & 2.6 & 0.6 & 18 & Negative \\
\hline & June 22, 2016 & Unchecked & Unchecked & 32 & Positive \\
\hline & July 2, 2016 & Unchecked & Unchecked & 28 & Positive \\
\hline
\end{tabular}

${ }^{\mathrm{a}}$ Reference ranges: Insulin, 2.7-24.9 $\mu \mathrm{IU} / \mathrm{mL}$; C-peptide, 0.7-1.9 ng/mL.

poor cross-reactivity to detect most insulin analogs (< $20 \%)$.

On the other hand, other assays, such as Mercodia IsoInsulin ELISA and Abbott Architect assay, have shown good potentials in detecting both human insulin and most insulin analogs ( $>80 \%$ cross-reactivity). The higher the complexity of an insulin analog is, the more difficult it will be to detect it (6). When surreptitious use of insulin is suspected, validated quantitative methods based on MS are the optimal choice for detecting insulin analogs (6). However, these methods are not available in Iran.

In the present case, we used Roche Elecsys insulin assay. Therefore, a high insulin level was reported when the patient used glyburide (detection of endogenous insulin), whereas a low insulin level was reported when he received insulin analogs. Several methods have been developed for the detection of antidiabetic drugs in the human plasma and urine. Some of these methods include micellar electrokinetic capillary chromatography, highperformance liquid chromatography (HPLC) with ultraviolet detection, HPLC with evaporative light scattering detection, and HPLC with charged aerosol detection $(7,9)$. The problem with most of these methods is their timeconsuming design and possibility of false positive results (7).

Introduction of LC-MS has attracted researchers' attention to this tool for detecting antidiabetic drugs in the plasma and urine. Chunyan Zhou et al. recently developed an LC-MS/MS method with a relative standard deviation of $<6.0 \%$, recovery rate of $83 \%-110 \%$, and analysis time of almost 8 minutes per sample (10).

It is important to determine the accuracy of these methods under optimal laboratory conditions. In our case, we sent the patient's serum and urine samples to a clinical laboratory in Iran, where the LC-MS/MS method is used for sulfonylurea detection. Despite high insulin and C-peptide levels in 2 serum and urine samples (samples 1 and 3; Table 1), sulfonylureas were negative. However, the results turned positive after several checks (samples 5 and 6; Table 1), which may be due to the lower drug concentration in the samples (lower than the sensitivity limit of the method).

As LC-MS/MS has been only recently applied in Iran for the detection of antidiabetic drugs, laboratory error is another possible explanation considering the manual design and complexity of this technique (11). As in our case, these laboratory defects can lead to the misdiagnosis of surreptitious use of insulin secretagogues as insulinoma or misdiagnosis of analog insulin use as noninsulin-mediated hypoglycemia. Due to the lack of any definitive laboratory findings, clinical suspicion is the best strategy for diagnosis (3). Detailed history-taking from all family members, including the patient's job, medical training, and conflicts, is essential to disclose any clues to factitious hypoglycemia.

Psychiatric factors are also important in these cases. Since factitious hypoglycemia is a serious psychiatric disorder, psychiatric consultation is an integral part of medical management. Psychiatric consultation is necessary not only for confronting the patient, but also for planning the subsequent treatments and follow-ups (1). However, the long-term outcomes of these patients are unpredictable. In a previous study, in which 10 patients with factitious hypoglycemia were followed-up for 15 years, only 3 returned to a productive life and 2 committed suicide (1).

\subsection{Conclusion}

It is suggested to suspect factitious hypoglycemia in patients with diverse biochemical patterns during different hypoglycemic episodes. Moreover, use of high-quality detection methods and platforms by experienced staff for the detection of antidiabetic drugs and insulin is suggested. Intensive psychiatric therapy and suicide prevention strategies are also necessary in these cases. Our patient was a unique case, as he was consuming insulin analogs and glyburide intermittently, resulting in confusing biochemical patterns. 


\section{References}

1. Grunberger G, Weiner JL, Silverman R, Taylor S, Gorden P. Factitious hypoglycemia due to surreptitious administration of insulin. Diagnosis, treatment, and long-term follow-up. Ann Intern Med. 1988;108(2):252-7. [PubMed: 3277509].

2. Walfish PG, Kashyap RP, Greenstein S. Sulfonylurea-induced factitious hypoglycemia in a nondiabetic nurse. Can Med Assoc J.1975;112(1):71-2. [PubMed: 803250].

3. Hirshberg B, Skarulis MC, Pucino F, Csako G, Brennan R, Gorden P. Repaglinide-induced factitious hypoglycemia.JClin Endocrinol Metab. 2001;86(2):475-7. doi: 10.1210/jcem.86.2.7160. [PubMed: 11157993].

4. Jordan RM, Kammer H, Riddle MR. Sulfonylurea-induced factitious hypoglycemia. A growing problem. Arch Intern Med. 1977;137(3):390-3. [PubMed: 190959].

5. Loh TP, Mok SF, Kao SL, Khoo E, Thai AC. Recurrent nocturnal hypoglycemia in a patient with type 1 diabetes mellitus. Clin Chem. 2014;60(10):1267-70. doi: 10.1373/clinchem.2013.214676. [PubMed: 25267512].

6. Parfitt C, Church D, Armston A, Couchman L, Evans C, Wark G, et al. Commercial insulin immunoassays fail to detect commonly prescribed insulin analogues. Clin Biochem. 2015;48(18):1354-7. doi: 10.1016/j.clinbiochem.2015.07.017. [PubMed: 26171976]
7. Giurgea I, Ulinski T, Touati G, Sempoux C, Mochel F, Brunelle F, et al. Factitious hyperinsulinism leading to pancreatectomy: severe forms of Munchausen syndrome by proxy. Pediatrics. 2005;116(1):145-8. doi: 10.1542/peds.2004-2331. [PubMed: 15995015].

8. Heald AH, Bhattacharya B, Cooper H, Ullah A, McCulloch A, Smellie $S$, et al. Most commercial insulin assays fail to detect recombinant insulin analogues. Ann Clin Biochem. 2006;43(Pt 4):306-8. doi: 10.1258/000456306777695690. [PubMed:16824282].

9. Shaodong J, Lee WJ, Ee JW, Park JH, Kwon SW, Lee J. Comparison of ultraviolet detection, evaporative light scattering detection and charged aerosol detection methods for liquidchromatographic determination of anti-diabetic drugs. J Pharm Biomed Anal. 2010;51(4):973-8. doi: 10.1016/j.jpba.2009.10.019. [PubMed: 20004074].

10. Zhou C, Tang B, Xi C, Zhang L, Wang G, Xi J, et al. Simultaneous determination of seven sulfonylurea type oral anti diabetic agents in adulterated dietary supplements and traditional chinese medicines by ultraperformance liquid chromatography tandem mass spectrometry. Spectrosc Lett. 2014;48(3):163-9. doi: 10.1080/00387010.2013.866965.

11. Grebe SK, Singh RJ. LC-MS/MS in the clinical laboratory, where to from here?. Clin Biochem Rev. 2011;32(1):5-31. [PubMed: 21451775]. 